\title{
Gradhiva
}

\section{La chorégraphie du bâton divinatoire comme écriture sonore au Burkina Faso}

The dancing choreography of the divinatory stick as acoustic writing in Burkina Faso

\section{Danouta Liberski-Bagnoud}

\section{OpenEdition}

\section{Journals}

\section{Édition électronique}

URL : http://journals.openedition.org/gradhiva/2367

DOI : 10.4000/gradhiva.2367

ISSN : 1760-849X

\section{Éditeur}

Musée du quai Branly Jacques Chirac

Édition imprimée

Date de publication : 16 mai 2012

Pagination : 182-201

ISBN : 978-2-35744-047-0

ISSN : 0764-8928

\section{Référence électronique}

Danouta Liberski-Bagnoud, «La chorégraphie du bâton divinatoire comme écriture sonore au Burkina Faso », Gradhiva [En ligne], 15 | 2012, mis en ligne le 16 mai 2015, consulté le 01 mai 2019. URL http://journals.openedition.org/gradhiva/2367 ; DOI : 10.4000/gradhiva.2367 

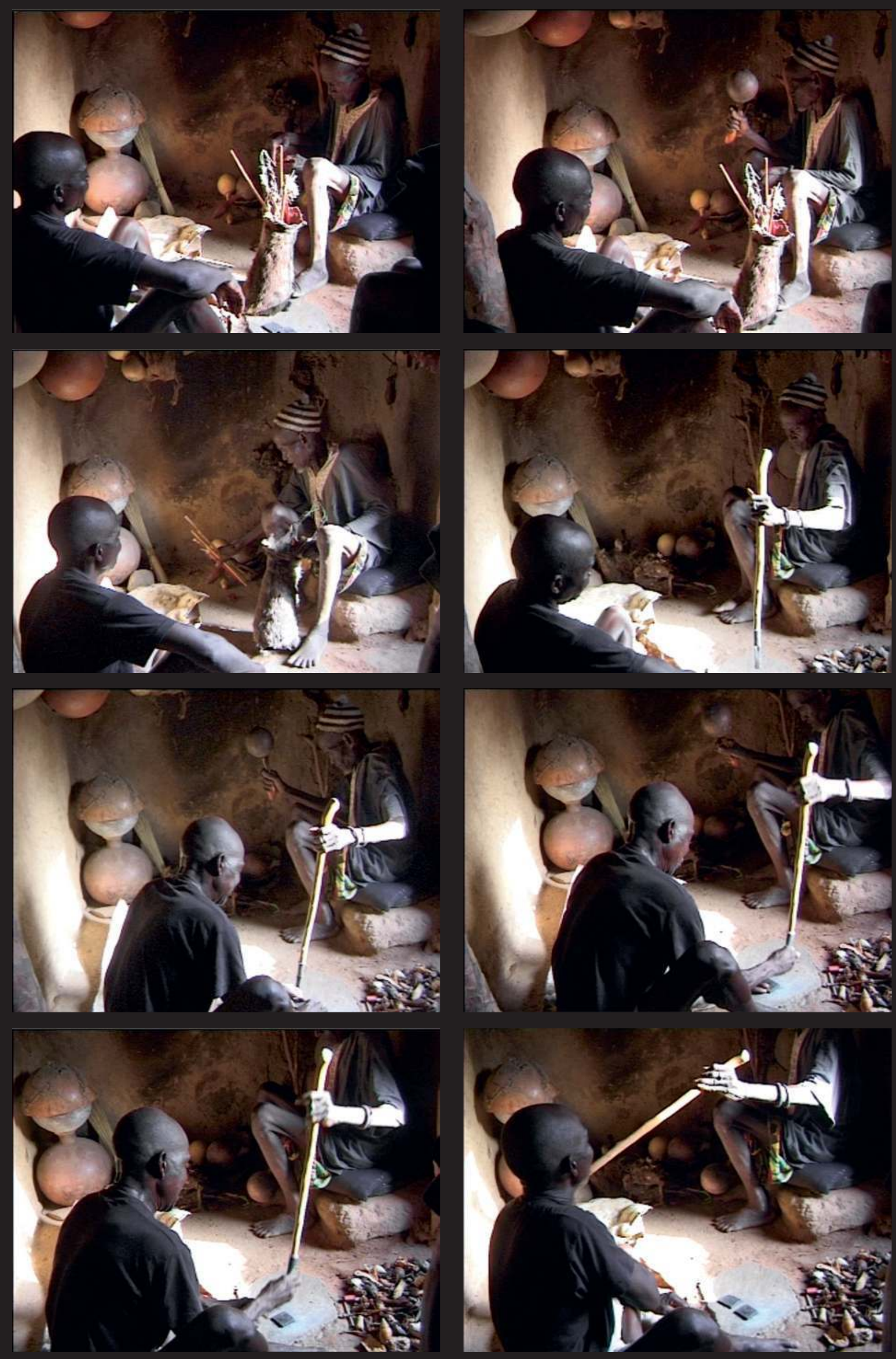


\section{La chorégraphie du bâton divinatoire comme écriture sonore au Burkina Faso}

\section{Danouta Liberski-Bagnoud}

Fig. 1 En ouverture, le devin se livre à un récitatif mi-parlé mi-chanté, dans le temps même où il met en place et articule les uns aux autres les différents éléments du dispositif oraculaire. Photogrammes du film Kaya-Navio,

Danouta Liberski-Bagnoud, 1998.

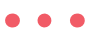

1. Citons l'ouvrage pionnier de Robert Jaulin (1966) sur les contraintes formelles du système géomantique et l'ouvrage d'Alfred Adler et Andras Zempleni sur la divination par les cailloux des Moundang (1972).

2. Michel Cartry, séminaire de l'École pratique des hautes études, 4 décembre 2008.
Il y a quelques décennies, Anne Retel-Laurentin, médecin et ethnologue, a soutenu que les systèmes divinatoires en vigueur au sud du Sahara, malgré l'extrême diversité de leurs techniques, tendaient tous à privilégier un mode de consultation de l'oracle où la part prise par le travail de lecture de signes était relativement secondaire par rapport à celle qu'occupait l'expression orale sous différentes formes et, en particulier, à celle qui revenait au travail de mise en mots des questions à poser ou des réponses à fournir (Retel-Laurentin 1974 : 295). De la place d'où elle fut professée - l'ouvrage Divination et rationalité, conduit sous la direction de Jean-Pierre Vernant-, une telle affirmation ne pouvait manquer de marquer durablement les études sur la divination dans les sociétés subsahariennes. À la même époque, des travaux décisifs ${ }^{1}$ avaient pourtant déjà montré, à propos de divinations de type géomantique, que ces techniques mobilisaient «des sémiologies complexes, où les paroles qui se disent et s'échangent au cours d'une séance sont étroitement tributaires de ce qui est $l u^{2} »$. Par ailleurs, fait curieux et persistant, la littérature consacrée aux divinations africaines fournit en réalité très peu de documents qui détaillent la place exacte qu'occupe la parole dans le procès de consultation de l'oracle. De telle sorte que la question du processus de production d'un énoncé oraculaire, qui est toujours un entrelacement de gestes et de paroles, conserve, dans la majorité des descriptions, sa part d'énigme, son statut de chose impensée et impensable; comme si les observateurs avaient été tout à la fois obnubilés par la technique et, d'une certaine manière, indifférents aux effets de sens qu'elle pouvait porter; comme si, peuton penser, ils étaient restés intimement persuadés que le discours du devin relevait, en fin de compte, d'une tout autre logique. Dans deux textes récents (2005 et 2009), qui marquent les derniers jalons de la recherche de longue haleine que Michel Cartry a consacrée à la géomancie gourmantché, cet auteur a apporté la preuve de la fécondité d'une approche qui, bien loin de poser l'existence a priori d'une dichotomie entre les gestes techniques et la parole, cherche à éclairer leur 


\section{-}

3. Cet article est fondé sur des données rassemblées entre 1982 et 1998 dans la province de Nahouri (Burkina Faso) auprès d'une dizaine de devins de langue kasem. Retournée récemment dans la région (2006. 2008), j'observe qu'en dépit des changements considérables venus modifier l'existence des villageois (école, dispensaire, route, téléphone portable, radio, vidéoclub), cet art divinatoire est loin d'être en voie de désaffection. Il a été transmis à une nouvelle génération de consultants, les enfants d'hier devenus «maîtres de maison », qui y cherchent toujours réponse à leur questionnement sur la conduite à suivre face aux aléas de l'existence.

4. Je reprendrai dans les pages qui suivent des éléments que j'ai déjà eu l'occasion d'exposer dans deux articles récents, l'un qui portait sur la sorte de théâtre de l'énonciation que met en place tout système divinatoire (2010), l'autre sur le statut ambivalent, à la fois ordures et choses sacrées, des signes visuels que manipule le devin par le bâton (2011).

5. Désormais, son corps a pour limites celles de la besace en peau qui contient la matière oraculaire (cf.infra). Si elle vient à brûler, son propre corps se couvre de pustules. J'ai exposé en détail le statut singulier du devin kasena dans un article précédent (2010: 124). rapport d'implication mutuelle. Il montre que "l'acheminement d'une parole divinatoire s'opère selon une ligne sans solution de continuité entre les procédures discursives et les techniques divinatoires mises en œuvre, depuis l'instant initial où le client murmure sa question [...] jusqu'au moment où, ayant fini de tracer la dernière figure du thème géomantique, le devin s'applique à mettre en mots ce qu'il est en train de lire en ces signes muets " (Cartry 2009 : 317). Le présent propos s'inscrit dans le droit fil du questionnement ouvert par cet auteur.

Au centre de la boucle du Niger, dans les pays qui ont en partage le bassin de la Volta, la divination dite "par le bâton " est l'un des modes de consultation de l'oracle les plus largement diffusés, et probablement l'un des plus anciens qui coexistent dans la région. Cette divination présente de nombreuses variantes, mais, en chacune d'elles, le dispositif technique sollicité est, en réalité, beaucoup moins élémentaire que ce que sa dénomination peut laisser croire. C'est à l'une de ces variantes locales, étudiée auprès de devins kasena du sud du Burkina Faso $^{3}$, que je vais ici m'attacher car elle offre un poste d'observation privilégié pour comprendre de quelle manière cet art divinatoire, qui mobilise un système de perceptions visuelles et sonores, met en branle une forme d' "écriture en acte " ainsi que des faits de lecture qui précèdent, déclenchent et guident la parole. Il ne s'agit pas cette fois de celle du devin comme dans la géomancie, mais de celle du consultant qui, tour à tour, questionne l'oracle et met en mots les réponses. Je préciserai plus bas ce que j'entends par "écriture en acte", mais afin d'éviter le caractère toujours un peu arbitraire d'une définition ex abrupto, quelques premiers éléments d'ethnographie doivent être d'abord donnés sur la forme générale que prend une consultation de l'oracle par le bâton chez les Kasena.

La divination occupe dans la vie quotidienne et rituelle des communautés villageoises kasena une place centrale. Qu'il s'agisse de guider l'action familière ou cérémonielle, individuelle ou collective - des funérailles à exécuter, la recherche d'un site pour habiter, un voyage à entreprendre, une naissance, une décision politique à prendre -, la consultation de l'oracle s'impose. Plongé dans le doute sur la conduite à tenir, le consultant cherche à clarifier les choix, à repérer des obstacles, à réparer des oublis - obstacles et oublis qui sont forcément en rapport avec ce monde de l'invisible, celui des ancêtres, des dieux du territoire, de l'instance Terre, des "médecines", etc., toute cette architecture cachée, immergée, que l'observateur occidental a tant de mal à saisir avec sa raison raisonnante. "Chercher à savoir où l'on en est dans ses rapports avec les dieux": cette formule, que j'emprunte, en la modifiant un peu, à l'assyriologue Jean-Marie Durand (2008: 568), pourrait parfaitement convenir à décrire ce qu'en son for intérieur un maître de maison kasena est susceptible d'énoncer pour lui-même alors qu'il se rend chez un devin. Le plus souvent, la consultation débouche sur la prescription d'une conduite rituelle à observer, d'un sacrifice à effectuer. Le questionnement de l'oracle est ainsi un réquisit de l'acte rituel, elle en fait intégralement partie, elle est de même nature (Cartry 1988 : 296). La divination n'est pas une simple activité, c'est une institution qui, avec la chefferie et la maîtrise de la terre, forme l'un des trois piliers sur lesquels repose l'organisation de la vie collective et individuelle des Kasena. Pour autant, le devin n'occupe pas une position sociale enviée. Il n'a pas choisi sa fonction et ne peut s'y dérober. Celle-ci se transmet le long des lignées, paternelle comme maternelle, la puissance "choisissant » l'héritier, qu'elle enlace si étroitement qu'il en perd les limites de son corps propres. Cette étreinte jalouse de la puissance - c'est «le temps de l'amitié amère », disent 
les devins - provoque des désordres considérables dans son existence la plus quotidienne jusqu'à ce qu'il accepte, contre son gré, la charge de devin (un statut qu'il n'acquiert qu'après une initiation). Jusqu'au terme de son existence, il devra assumer les exigences de la charge. À tout moment de la journée, si un client l'appelle, il doit interrompre son activité, retourner chez lui et procéder à l'ouverture d'une séance de consultation. On ne peut surseoir à une demande de l'oracle. De cette tâche incessante, il ne tire quasiment aucun bénéfice : les petits dons (une noix de cola, des fragments de tabac, quelques piécettes de monnaie) offerts par le consultant pour «solliciter la bouche de l'oracle" reviennent en grande partie à la puissance oraculaire, sous la forme d'animaux sacrifiés à intervalles réguliers. On retiendra enfin que le personnage ne possède aucun de ces traits sous lesquels sont dépeintes certaines figures de devins africains : il n'est ni un thérapeute, ni un vecteur du contrôle social. Son rôle, on le verra mieux plus loin, est celui d'un intercesseur : partageant désormais le même espace-corps que la puissance, il a la capacité d'inviter celle-ci à répondre aux questions du consultant et de procurer ainsi à ce dernier l'accès à une parole jugée vraie - un statut qui puise sa garantie dans le montage de gestes et de discours, réitéré au début de chaque consultation, qui établit la maison de Soleil-Dieu comme lieu tiers d'où proviennent toutes les paroles de l'oracle.

Tout le monde peut se rendre auprès de l'oracle, sans considération de statut, d'âge ou de sexe. Les plus expérimentés dans le maniement du bâton (cf.infra) se rendent seuls chez le devin, les novices demandent à un proche parent, parfois un ami intime, de les y accompagner. On n'expose jamais les motifs pour lesquels on s'est rendu chez le devin; le consultant introduit sa demande en la coulant dans une formule fixée par la tradition dont les premiers mots sont: "Je suis venu demander ta bouche pour que tu me dises quelle est la chose qui déloge qui m'a expulsé hors de chez moi. " Autrement dit, il demande à l'oracle de lui montrer ce qui l'a contraint à sortir de chez lui pour venir le questionner. Il est difficile de formuler de façon plus opaque le fond de la demande. Plantés face à face, le devin assis sur une banquette en terre, surplombant de peu le consultant qui est installé sur une peau de bœuf étalée au sol, ils tiennent chacun l'une des extrémités de la canne divinatoire - un simple bâton de bois patiné par l'usage qui s'achève, d'un côté, par une fourche que tient le devin et, de l'autre, par un embout ferré que le consultant retient entre le pouce et l'index. Comme soudés l'un à l'autre par cette canne, ils vont se livrer pendant toute la durée de la consultation à un étonnant ballet, coordonnant sans heurts leurs mouvements, donnant à ceux qui les observent l'impression de suivre, et non de guider, les déplacements rapides et continus du bâton. Hors la canne et ses mouvements, la " matière oraculaire ${ }^{6}$ " est fournie par une collection hétéroclite d'objets fabriqués et de choses prélevées à fleur du monde dont le rassemblement dans une besace en peau constitue la somme de "tout ce qui existe sur terre". Ces choses, ainsi extraites de la réalité qu'elles auront à signifier, composent un lexique rudimentaire au sein duquel le bâton va sélectionner les autres mots de l'oracle; les objets choisis sont déplacés et laissés en un coin de la "table " de consultation - le morceau de sol où sont disposés certains des éléments de l'appareil divinatoire. Ils vont y constituer une sorte d'archivage temporaire des différentes "paroles" de l'oracle au cours d'une même séance et viendront soutenir la mémoire du consultant quand, en une ultime mise à l'épreuve de la véracité du «dit» oraculaire, il devra finalement récapituler l'ensemble des prédictions qui lui ont été dévoilées.
-

6. Dans son étude de 1966 sur la divination par le bâton chez les Talensi (nord du Ghana), Meyer Fortes introduit le concept de materia oraculosa pour désigner le contenu du sac, qu'il décrit par ailleurs comme «a lot of odds and ends of rubbish » (198? [1966] : 15]. Les sacs des devins talensi et kasena accumulent principalement des ossements et autres parties d'animaux domestiques et sauvages, divers débris du monde végétal [cultivé et naturel), quelques extraits du monde minéral (fer, terre, sel) et de nombreux fragments d'objets fabriqués. 
ÉTUDES ET ESSAIS

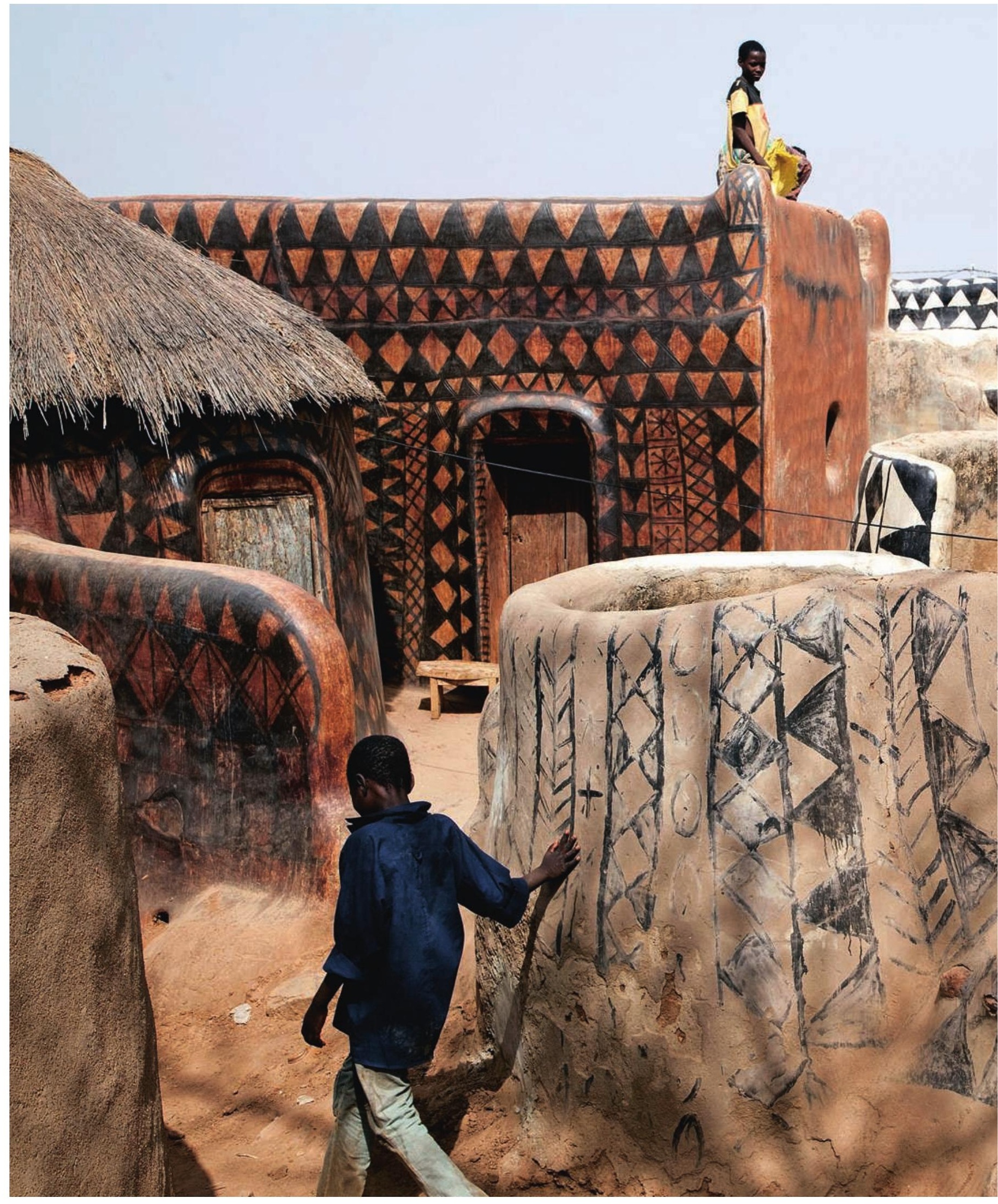


- -

7. Meyer Fortes (1987 [1966]), Robert Sutherland Rattray (1969 [1932]) et Eugene Mendonsa (1982).

8. Rattray, plus préoccupé de rendre compte de la place essentielle qu'occupe ce type de divination dans la vie rituelle des peuples de l'arrière-pays ashanti, ne s'engagera jamais sur cette pente.

9. La notion ne doit pas être confondue avec les «actes d'écriture » qu'analyse avec beaucoup de subtilité Béatrice Fraenkel [2007). La direction prise ici ne s'inscrit pas dans le domaine de l'anthropologie de l'écriture qu'elle promeut, bien que, forcément, des lieux de croisement ne peuvent manquer d'exister.

10. Cf. également l'excellent article qu'Anne Boissière consacre à Schaeffner et aux origines corporelles de l'instrument de musique (2011).
Les rares auteurs qui ont eu à répondre à la question de savoir comment «ça parle " se sont attachés, pour l'essentiel, à rendre compte de ce qui relève du code symbolique qui assigne à chaque objet un ou plusieurs signifiés? ${ }^{7}$ Dans cette perspective, la divination est appréhendée comme une technique de communication entre les hommes et les dieux; le devin est en position de déchiffreur et son client doit monnayer le sens minimaliste et générique de la sentence oraculaire en une prescription détaillée de l'acte rituel qu'il aura à exécuter. Sans être inexacte ou erronée, cette approche a cependant le défaut de réduire la consultation de l'oracle à un pur acte (mental) de décryptage, et manque du même coup un point essentiel de discrimination, à savoir qu'ici tant le corps du devin que celui du consultant sont impliqués dans le procès de fabrication de l'énoncé oraculaire, qu'ils y sont tout entiers engagés. En faisant la part belle au code, tout en réduisant l'aléatoire à un jet de cauris en début de consultation, deux de ces auteurs butent par ailleurs sur ce qui semble constituer pour eux un embarras, à savoir le caractère "objectivement" arbitraire de la technique mise en œuvre - quoique "subjectivement vécue par les protagonistes comme rationnelle" (Fortes 1987 [1966] : 18); ce qui les amène nécessairement à faire planer sur ce mode de consultation divinatoire, sous la forme d'une dénégation pour Meyer Fortes, d'une affirmation pour Eugene Mendonsa, l'ombre du trucage et de la tromperie ${ }^{8}$. Question, on en conviendra, qui n'a d'autre intérêt que de révéler la croyance de celui qui se la pose.

Pour sortir de ces impasses, il convient de changer l'angle d'étude et d'examiner avec la plus grande minutie - au plus près des mots, des gestes et des objets qui entrent dans la composition de l'appareil divinatoire - le procès de production d'une parole qui, pour le consultant, fera effet de vérité. À cette échelle micrographique, on s'apercevra alors qu'avec la sorte de corps-à-corps auquel se livrent le devin et le consultant tout le long d'une séance divinatoire, ce qui s'accomplit, ce n'est pas tant la transposition en langage clair de signes obtus captés depuis le monde de l'invisible que l'acte de construction d'un texte porteur d'un sens dont ni le devin ni le consultant ne sont à proprement parler l'auteur. L'absence de tout système d'inscription durable, hors les traits aériens et fugitifs que trace le bâton dans les airs, semble exclure a priori de penser les formes du dire mises en œuvre dans ce type de divination sous le registre de l'écriture. La manière même de "questionner l'oracle», cette étrange gestuelle couplée du devin et du consultant qui paraissent exécuter une chorégraphie réglée par la canne même, conduit cependant à la rapprocher de ce que Roland Barthes a défini comme acte d'écriture ${ }^{9}$, c'est-à-dire l'écriture en train de se faire et non comme produit, l'écriture comme mouvement et non comme forme, et dont il souligne avec force qu'elle est une «expression du corps dans l'espace [...] attachée, non à la voix, mais à la main, au muscle [...] » (Barthes 2000 [1994] : 67sq.). À la suite d'André Leroi-Gourhan et sa magistrale leçon d'anthropologie sur le couple main-graphie et voix-audition (1964: 270 sq.et 290 sq.), il rappelle que «l'écriture est toujours du côté du geste, jamais du côté de la face : elle est tactile, non orale" (Barthes 2000 [1994] : 72). Cheminant sur la même voie, mais en un lieu où elle croise le sillon creusé par André Schaeffner et ses recherches sur le corps comme lieu matriciel des instruments de musique ${ }^{10}$ (1995 [1936]), Pierre Legendre nous fera faire un pas de plus en insistant sur le fait que «l'art d'écrire ne se limite pas au scriptural incrustant le dire dans des signes matérialisés, objets séparés du corps humain", mais se réalise encore "dans des assignations chorégraphiques, ou plus généralement théâtrales, transformant le corps lui-même en signe et écriture de signes 》 (2001 : 


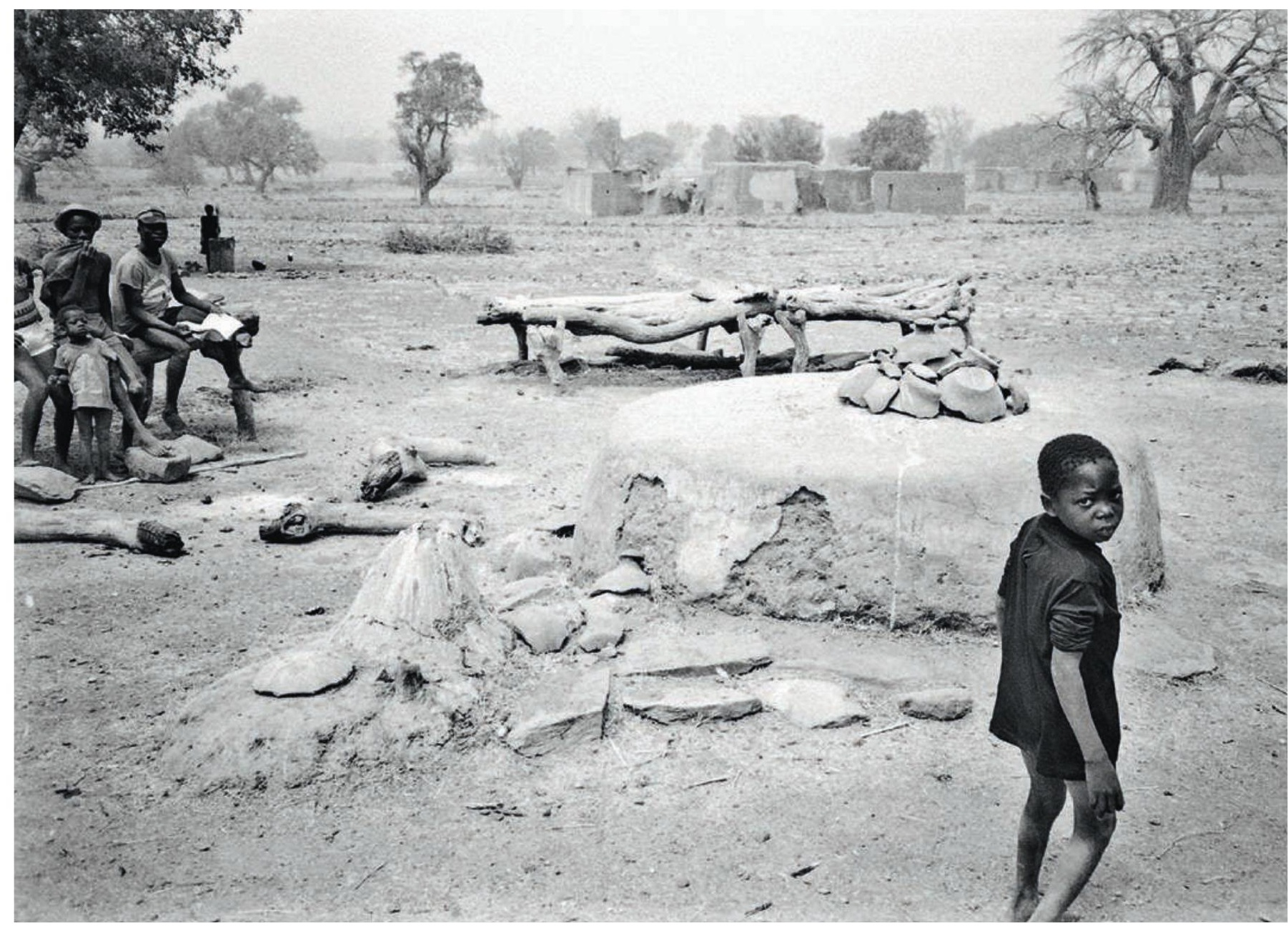

Fig. 3 Dans la cour extérieure d'un devin, l'autel de la divination fait d'un cône en banco et d'une poterie enterrée a été construit sur les flancs du grand autel des ancêtres lignagers du devin. Kaya Pungu, 1985, photo Danouta Liberski-Bagnoud.

220). Les sociétés sont orales, écrivaient Gilles Deleuze et Félix Guattari, «non pas parce qu'elles manquent d'un système graphique (une danse sur la terre, un dessin sur une paroi, une marque sur le corps sont un système graphique), mais parce que ce système graphique est indépendant de la voix, ne se subordonne pas à elle mais lui est connecté, coordonné "dans une organisation en quelque sorte rayonnante ${ }^{11 \text { ” }}$ et pluridimensionnelle » $(1972: 222)$. Les recherches les plus récentes sur l'histoire de l'écriture, sans aller jusqu'à délier complètement l'écriture de la voix - car c'est bien de s'être soumise à la parole que la main traçant des graphes s'est mise à écrire -, reconnaissent l'impasse où les tenants de la théorie selon laquelle l'écriture (alphabétique) représente la parole avaient jeté la discipline, et proposent l'idée que ce qui a fait événement dans l'apparition de l'écriture c'est qu'elle rend la parole visible (Christin 2001: 10). Ce double tour donné à la façon d'aborder l'écriture - comme système graphique non subordonné à la parole et à lire dans les gestes rythmés d'un corps dansant ${ }^{12}$ - permet de lever l'hypothèque qu'une tradition bien établie fait peser sur l'étude des divinations en maintenant une ligne de fracture entre les pratiques de type géomantique, qui sollicitent une «raison scripturaire», et les autres, qui relèveraient de la seule oralité. Les
-

11. Les auteurs citent ici Leroi-Gourhan (1964 : 273).

12. Un art d'écrire du corps dansant qui ne se confond pas avec, ni n'est tout entier consigné par le système de notation mis au point en Occident à l'aube du xvIII siècle pour transmettre les pas de danse, comme le souligne Legendre, mais qui est en lien étroit avec ce que Marcel Mauss, sous la rubrique « techniques du corps », a dévoilé de l'ouvrage de la raison pratique collective dans l'art d'utiliser le corps humain (Mauss 1980 [1950]: 369]. 


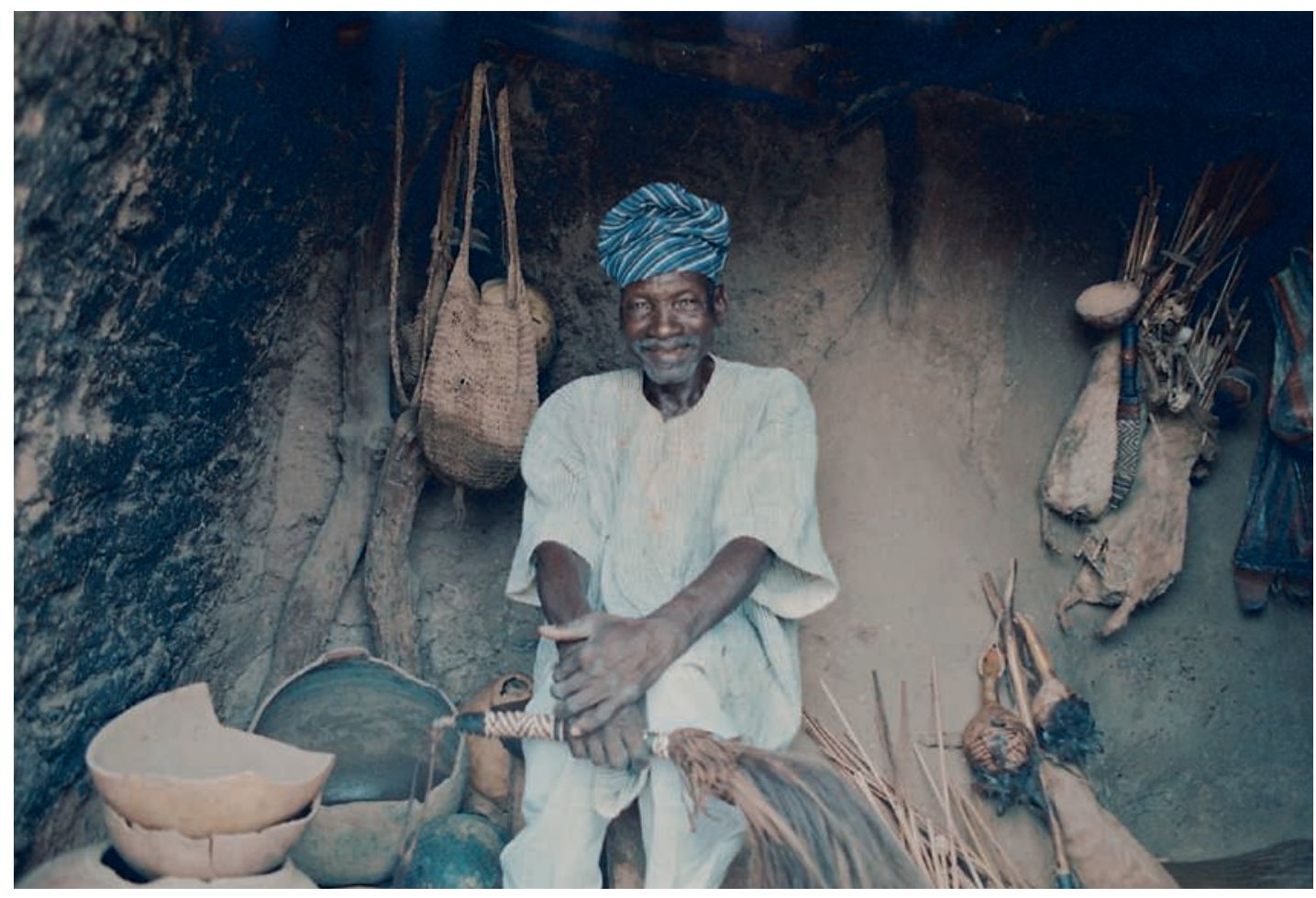

Fig. 4 Le devin Segungu Atibu, dans la petite pièce réservée aux consultations de l'oracle. À sa droite, reposant sur le sol, son sac piriforme contenant les «objets-mots » et la canne divinatoire supportant deux hochets calebasse. Accrochés au mur, d'anciens sacs hors d'usage. Kaya Navio, 1994, photo Danouta Liberski-Bagnoud.

unes comme les autres reposent sur un même mécanisme général selon lequel les signes (la figure géomantique, l'objet-mot), bien loin de s'aligner sur la voix comme l'écriture linéaire, commandent à la voix (du devin ou du consultant, en fonction des techniques divinatoires) et déclenchent la parole.

Qui assiste pour la première fois à une consultation de l'oracle par ce type de devin que les Kasena appellent vuru, que nous traduisons par «celui qui martèle », ne peut qu'être abasourdi par l'afflux de perceptions sonores et visuelles, par l'assaut de signifiants qui lui paraissent d'autant plus "purs» qu'il n'a ni l'oreille ni l'œil éduqués pour y déceler quoi que ce soit susceptible de faire sens pour lui. De cette première expérience, il ne pourra retenir qu'une idée générale de rapidité, de mouvements et de bruits. Toutes sortes de bruits. Le son métallique que produit l'entrechoquement répété de l'embout ferré de la canne divinatoire contre deux petites plaques en fer, le bruit plus sourd quand cette même canne vient frapper le sol, plus sonore quand elle vient heurter la large pierre sur laquelle reposent les plaquettes, mais aussi les notes aiguës que le devin tire de sa flûte ou encore le crépitement rythmé d'une sorte de maracas unique (une gourde calebasse évidée et emplie de graines) qu'il agite tout au long de la séance. Ce sont là autant de sonorités diverses qui viennent se superposer et souvent recouvrir les paroles claires du devin et du consultant, et plus encore leurs murmures ou le fredonnement auquel se cantonne le devin durant la quête du consultant, interrompant de temps à autre sa mélopée par quelques interjections répétitives du type «où allons-nous?», «tu as vu!», «je n’ai pas parlé! ». Le 
mouvement, lui, est créé par les déplacements incessants de la canne divinatoire que le devin et le consultant tiennent délicatement, sans avoir l'air d'y toucher. Donnant l'illusion d'être animée par une impulsion propre, la canne vient tantôt indiquer tel ou tel point de l'espace, telle ou telle partie du corps du consultant, tantôt fouiller le tas d'objets et de choses jetés en vrac au pied du devin pour en extraire un élément, tantôt encore frapper les plaques en fer en des séquences répétitives, visiblement selon un code bien établi.

Objet singulier dans le paysage des techniques divinatoires, ce langage oraculaire fait de sons et de mouvements ne se laisse ranger selon aucune des grandes dichotomies habituellement établies pour tenter d'introduire un début d'ordre dans le champ quasi infini des modes de consultation de l'oracle. On ne peut le classer ni tout entier du côté des divinations «inspirées" ou "spontanées", où le devin est censé entrer en communication directe avec la divinité qui rend l'oracle, ni tout entier du côté de ces divinations "artificielles", où l'oracle se fabrique pour l'essentiel à partir du déchiffrement par des spécialistes de signes naturels ou patiemment construits dans le cours même de la consultation, selon les règles de la raison graphique ou celles de la raison calculante. À supposer que cette distinction ait quelque valeur heuristique, il faudrait soutenir que la divination "par le bâton » est une combinaison de ces deux grands types. D'une part, si le personnage du devin peut à certains moments de la consultation, et notamment en son commencement, se dire (et être perçu comme) «inspiré " par ce qui, en cette forme de divination, est en place de puissance oraculaire ( «il l'entend et ne peut s'empêcher de dire ce qu'il entend »), il n'entre à aucun moment dans cet état que l'on qualifie de transe. Comme le faisait déjà remarquer Fortes à propos des devins talensi, ce type de personnage n'est jamais «dissocié » (au sens psychique du terme). En outre, l'usage qu'il fait de la parole se limite à un usage qu'on pourrait presque qualifier de «technique ». La longue incantation qu'il exécute d'une voix de tête à l'ouverture de la séance a pour visée explicite d'inviter les différentes composantes de l'instance oraculaire à se rassembler dans l'espace où se tient la consultation. Jamais le consultant n'interprétera la parole du devin, y compris en ce moment inaugural de la consultation, comme des prophéties directement dictées par l'oracle. Par ailleurs, bien que la technique mise en œuvre ne comporte aucune sorte de calcul ou d'inscription matérialisée, elle a recours à un langage artificiel avec son lexique et ses règles de formation des énoncés qu'on peut axiomatiser et qui se retrouvent, dans une population donnée, chez tous les praticiens de cette forme de divination.

Ce langage artificiel repose sur un ensemble de perceptions produites dans le temps même de la consultation, ensemble qui n'est pas d'une seule et même nature, sonore ou visuel, mais l'un et l'autre : les mouvements du bâton, les objets du sac autant que le heurt répété de la canne contre les palets en fer ou contre le caillou qui les supporte participent concurremment à la fabrication de l'énoncé oraculaire. La percussion est, ici, l'un des procédés constitutifs du langage de l'oracle; elle en serait même, selon la langue, le moyen d'expression princeps. En effet, le nom donné à cette forme de divination par le bâton, vugu, est formé sur la base verbale / $v u /$ qui désigne une modalité spécifique du " frapper ", celle que réalise précisément la canne divinatoire à certains moments de la consultation quand, par un mouvement régulier de haut en bas, elle vient heurter de son embout ferré les plaquettes de fer ${ }^{13}$. On apprendra plus loin que ce qui se joue en ces séquences où dominent les sonorités métalliques tirées de l'entrechoquement de la virole et des plaques est décisif pour l'établissement final de l'énoncé
- 0

13. Le radical verbal/vo/ est spécifique au domaine de la divination. La Société internationale de linguistique le traduit par « deviner, consulter le devin ». II m’apparaît mieux servi par le sens de «marteler » ou «dire l'oracle en martelant », car cela permet tout à la fois de restituer la dénotation kasem du terme (le mouvement percuteur de la canne) et de connoter le lacis de significations liées au monde de la forge qui enserre ce mode de questionnement de l'oracle. 


\section{- $\bullet$}

14. Sur la question difficile de l'introduction dans la technique divinatoire d'une certaine dose de ce que la raison scientifique détermine comme l'aléatoire, mais qui n'est pas perçu comme tel par les praticiens de la divination, on se reportera utilement au texte de Michel Cartry [2005].

15. Cette formule, qui lève un coin du voile sur la théorie endogène concernant la nature de la parole oraculaire, fait partie d'un ensemble discursif dont j'ai cherché à montrer ailleurs les différentes articulations (2010 et 2011). oraculaire. Retenons pour l'instant que les perceptions visuelles et sonores produites par les déplacements de la canne divinatoire ne sont pas en elles-mêmes des présages que doivent chercher à déchiffrer le devin et le consultant. Chacune d'elles forme plutôt un signifiant renvoyant à un ou plusieurs signifié(s) possible(s) et constitue l'unité minimale du langage de l'oracle. Dans le temps même où ces signifiants surgissent, le discours du consultant vise à les fixer comme signes, supports d'un signifié unique, insérés dans une trame signifiante, le « dit » de l'oracle, qu'il va pouvoir mettre à l'épreuve en recourant à plusieurs procédés de vérification.

L'une des questions majeures que soulève cette forme de divination est celle du statut respectif du devin et du consultant dans la production d'un énoncé oraculaire. Ces deux protagonistes de l'acte divinatoire ne peuvent en effet être différenciés en fonction de ces traits discriminants généralement présents dans les autres formes de divination, qui attribuent au devin un savoir propre, voire une forme d'inspiration, et placent le consultant dans le rôle d'un client sollicitant un spécialiste. Questionner l'oracle relève de compétences que tout homme, toute femme peut acquérir auprès d'un devin, par une démarche volontaire et sans autre obligation que celle de l'apprentissage par la pratique. Le consultant qui est passé maître dans l'art de questionner - « celui qui sait questionner » est le terme kasem qui le désigne - est en position d'interprète de ce langage de l'oracle, fait de gestes, d'objets et de sons; il en est même souvent le seul qui soit audible. Il ne se contente pas, toutefois, de mettre en mots les signifiants sonores et visuels pointés par la canne divinatoire, il concourt également avec le devin à leur production, à leur construction en signes et combinaison en phrases, et subséquemment à la composition finale du texte de l'oracle.

La technique divinatoire recourt à trois procédés distincts, tous trois constitutifs du langage oraculaire : le jet d'objets, un jeu de signifiants (les «choses » et les "gestes») et, enfin, les déplacements du bâton. Le premier de ces procédés s'appuie sur un principe fort répandu, la place qu'il laisse à ce que notre mode de pensée, caractérisé par l'emprise de la raison scientifique, définit comme le hasard étant une donnée récurrente de toute technique divinatoire ${ }^{14}$. Le "jet d'objets " n'intervient qu'une seule fois au cours d'une séance, avant que commence la consultation proprement dite. Arrivé aux deux tiers de son récitatif d'ouverture, le devin retourne la «bouche » du sac vers le sol et le secoue vigoureusement afin que les objets tombent en un tas plus ou moins dispersé. Cette gestuelle, que le devin accompagne d'une exhortation à l'oracle afin qu'il «vienne vite", est un moment décisif de la séance, celui où « vuru décharge de dessus sa tête les paroles issues de la maison de Dieu ${ }^{15}$ ». Observer la disposition des objets tombés au sol donne lieu à un temps de lecture propre, distinct du maniement de la canne et de la sélection des objets. Loin d'être un geste fonctionnel, cet acte du devin produit un premier jaillissement de la parole oraculaire: l'emplacement des objets par rapport à l'ensemble (sur les bords ou au sommet de l'amas, enfouis ou isolés), mais aussi les uns par rapport aux autres (côte à côte, en croix, l'un par-dessus l'autre), constitue autant d'indices qui, d'emblée, détachent du reste du tas une première configuration de signifiants à partir desquels le consultant va chercher à élaborer un premier questionnement. Le deuxième procédé utilisé repose également sur un principe (le code) largement récurrent en d'autres formes de divination, bien que sa réalisation présente ici quelques particularités. Les champs notionnels couverts par ce lexique sont étroitement ajustés aux fins d'une consultation de l'oracle. Une proportion importante d'objets (le tiers, 
parfois la moitié du contenu d'un sac) renvoie à l'un ou l'autre aspect de la pratique rituelle: des types de rites, mais aussi les différentes entités susceptibles d'être mobilisées par le système sacrificiel, des modalités d'offrandes et de sacrifices ou encore des catégories d'autels d'ancêtre. Lorsqu'il questionne l'oracle, un consultant kasena ne cherche pas tant en effet à obtenir un savoir sur l'avenir qu'à dévoiler ce qui, dissimulé sous les événements du présent, est susceptible d'en décider l'issue et ce qu'il est en son pouvoir de faire pour que cela tourne à son avantage. Ces «choses cachées " peuvent être tantôt un rite en souffrance, tantôt des dettes sacrificielles oubliées ou ignorées que lui ou l'un de ses ascendants a pu contracter auprès de l'une des catégories d'instances ou de puissances (ancêtre, dieu du territoire, "médecine", fétiche) qui donnent à l'invisible sa configuration propre. Toute consultation de l'oracle se traduit donc in fine par un certain nombre de prescriptions sacrificielles, et le vocabulaire en objets dont dispose l'oracle répond à cette exigence.

Le troisième procédé, qui exploite les lois de la cinématique, est en revanche moins répandu. Il nous intéresse tout particulièrement car il partage avec l'acte d'écriture (sa praxie) une manière propre d'engager le corps dans la production de graphes signifiants. Par le geste d'agrippement et de pression digitale exercé sur l'instrument d'écriture, le corps du scripteur est tout entier impliqué : dans le tracé opéré peuvent se lire, a posteriori, certaines caractéristiques physiques de l'acte, telles que la tension et la pression du trait, et, par là même, s'y révèlent

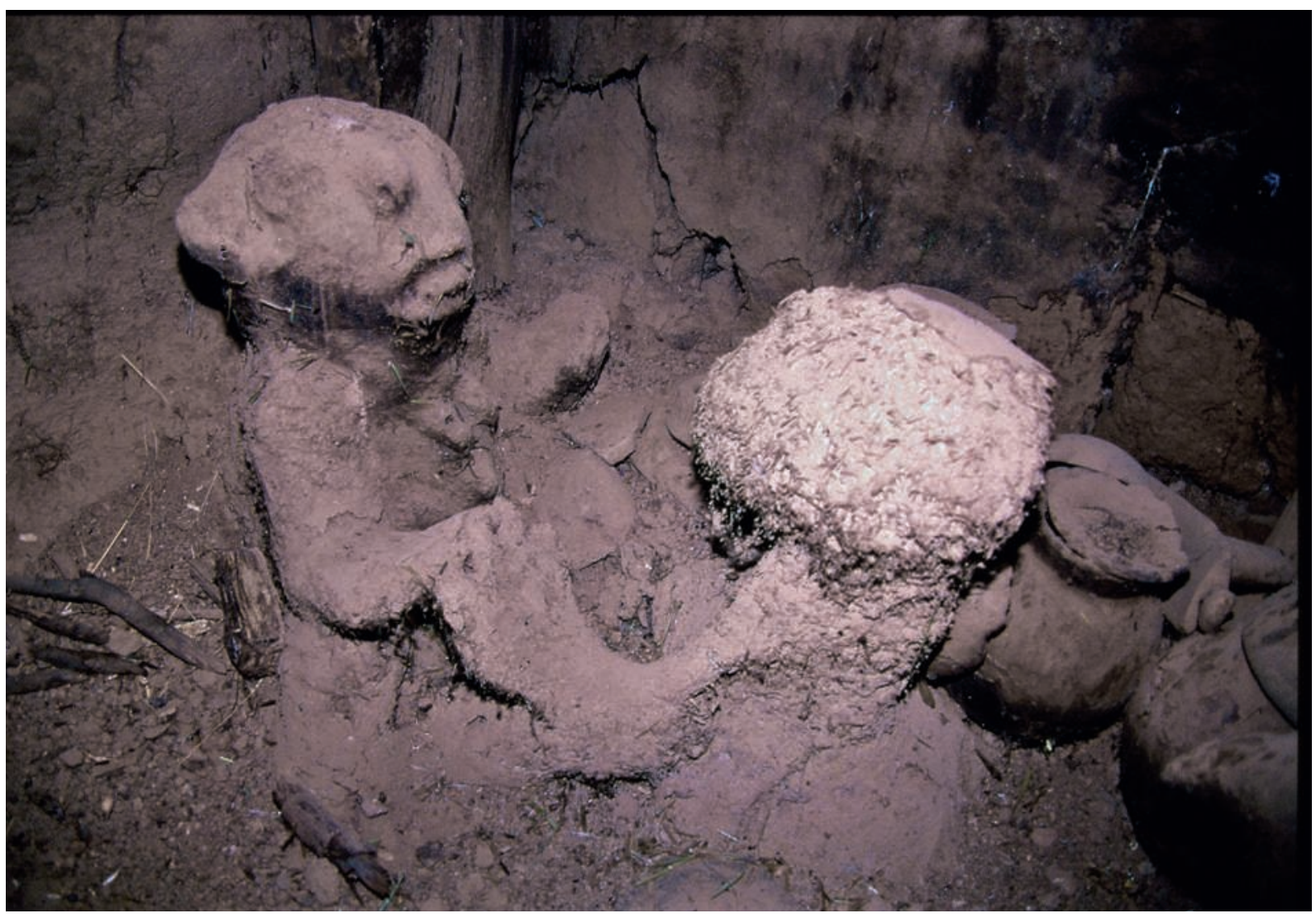

Fig. 5 Couple de statues en terre pétrie figurant une consultation de divination. Présentification de la puissance ancestrale d'un devin dagara dont la lignée remonte à un grand devin issu de Kasana, un village kasena situé au Nord du Ghana. Legmoin, 1985, photo Daniela Bognolo. 


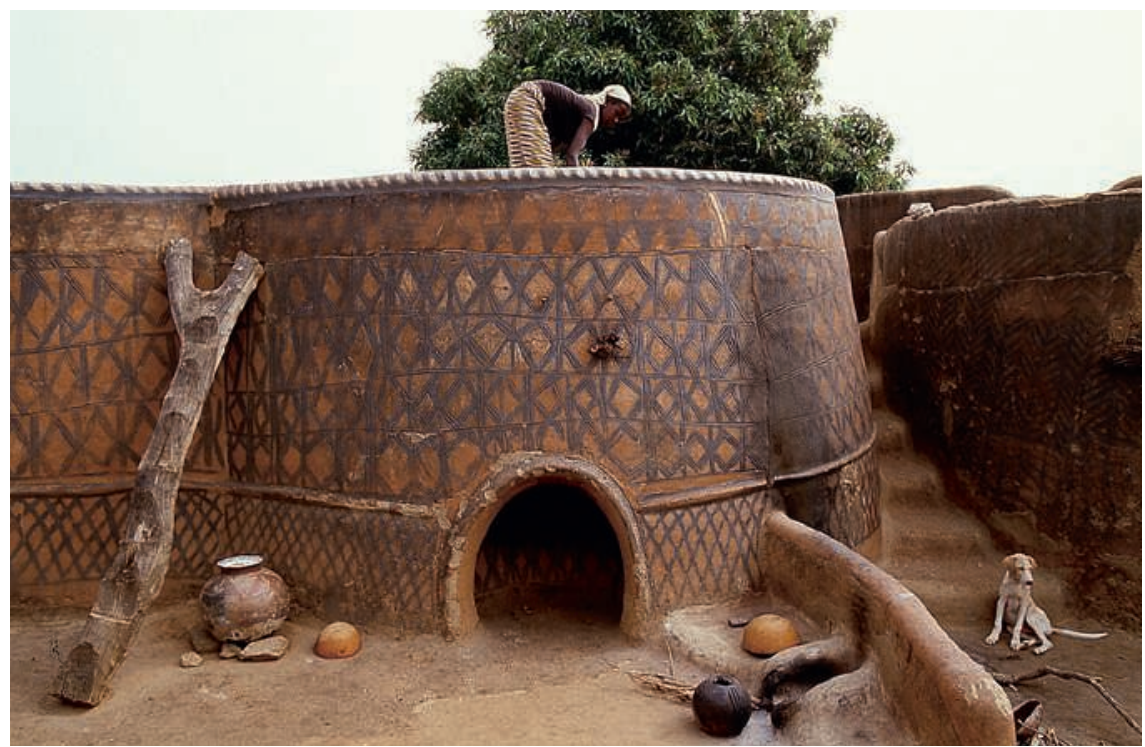

Fig. 6 Les fresques que les femmes kasena peignent sur les parois de leurs appartements sont composées d'un ensemble ouvert de signes. Un très petit nombre d'entre eux sont communs à ceux tracés à la pointe de la canne divinatoire. Korso, 1987, photo Danouta Liberski-Bagnoud.

ce que Barthes appelait les "profondeurs pulsionnelles du corps» (2000 [1994] : 26). Dans la technique dite du bâton, la seule prise asymétrique de la canne divinatoire - de la main gauche pour le devin, de la droite pour le consultant provoque d'infimes mouvements, imperceptibles à l'œil pour un observateur, chaque tenant du couple exerçant une pression de saisie particulière, hors de toute volonté consciente. Ces infimes oscillations sont ressenties de façon kinesthésique par les deux protagonistes et, voulant les accompagner car elles sont perçues par eux comme le mode du dire propre à l'oracle, ils sont amenés à les amplifier, faisant décrire à la canne de larges mouvements perceptibles par tous.

Les mouvements de la canne divinatoire ne sont pas tous au service d'une même fonction. Certains assurent la sélection des «mots» de l'oracle (objets et gestes), d'autres permettent de produire une assertion, d'autres enfin viennent séparer les différentes phases dans la fabrication d'un énoncé oraculaire ou encore distinguer les différentes parties d'une consultation, véritables ponctuations qui ordonnent et structurent le flux du questionnement. La fonction de sélection n'appelle pas de longs commentaires. Le mode en est assez simple : la partie ferrée de la canne pointe ou heurte légèrement l'élément qui devient ipso facto une unité minimale de l'énoncé oraculaire. Il peut s'agir d'une chose provenant du tas d'objets naturels et façonnés qui fournit des lexies simples (mots) et complexes (locutions, syntagmes). Il peut s'agir également d'une partie du corps du consultant, d'une direction de l'espace ou encore d'éléments de la pièce (sol, mur, siège du devin, etc.). L'utilisation de la canne divinatoire pour établir une proposition jugée vraie est plus complexe. Elle requiert le jeu des petites plaques en fer et de la virole de la canne, constituant un rouage essentiel de la «machine à parler » qui permet de produire in fine une assertion, c'est-à-dire une proposition qui, dans sa forme, peut être affirmative ou négative et que l'on soutient comme vraie. Après s'être assuré que les différents signifiants sélectionnés par la canne dans la phase antérieure font bien partie de la configuration à questionner (nous y reviendrons plus loin), le consultant insère un premier item (par exemple 
« mon père ») dans un système à deux propositions dont l'une est nécessairement une affirmation et l'autre une négation, créant ainsi une alternative positive/ négative (ou négative/positive). Soit, dans l'exemple choisi, la construction d'une alternative du type « mon père est présent »/« mon père est absent », plus généralement formulée sous la forme interrogative. Dans le temps même où il crée cette alternative, le questionneur lie respectivement chaque proposition (affirmation et négation) à l'une des deux plaquettes en fer (en pointant celle-ci du doigt ou en la soulevant légèrement pour la claquer aussitôt contre le sol). Aussitôt fait, il procède à une forme de tirage au sort en se servant de la canne, toujours tenue à l'autre extrémité par le devin. Le heurt, à trois reprises, de la virole contre l'une des deux plaquettes, et seulement celle-là, sélectionne la réponse à sa question (qu'il s'agisse de la proposition affirmative ou négative). Le consultant réitère par trois fois la même procédure en veillant, à chaque tirage, à inverser les attributions de sens aux plaquettes de métal, à formuler sa question de façon inaudible ou encore à ne la formuler qu'en pensée.

Enfin, il est un dernier usage de la canne qui, par une combinaison stable de mouvements jointe à l'énonciation d'une même formule ("c'est cela que j'ai vu»; "c'est cela que tu as dit»; "quoi d'autre?/je n'ai pas parlé »; "nous acceptons»), offre les rudiments d'une ponctuation qui permet de démarquer différents moments dans le processus d'élaboration d'un "dit» de l'oracle, ainsi que différentes phases dans le déroulement d'une consultation. On repère quatre de ces combinaisons de gestes, de heurts et de mots qui viennent scander le flux de la parole oraculaire, sortes d'équivalents lointains de ces respirations de l'écriture que sont la virgule, le point-virgule, le point d'interrogation et le point final. Dans la mesure où ces démarcatifs, par l'ordonnancement de la parole qu'ils provoquent, introduisent des divisions dans le texte oraculaire, établissent des rapports entre celles-ci et permettent ainsi que du sens surgisse, on est en droit d'en parler comme d'une sorte de syntaxe.

Les déplacements de la canne, dans les trois fonctions qu'ils remplissent (lexicale, assertorique, syntaxique), sont toujours une translation des impulsions qui lui sont données, lesquelles, selon notre point de vue, résultent des pressions contradictoires des deux "scripteurs", mais qui, selon le point de vue des praticiens de cet art, doivent être imputées à vogu, l'oracle. "La canne, expliquent les devins interrogés, est tenue par la puissance oraculaire, c'est pourquoi devin et consultant doivent la prendre délicatement, pour ne pas forcer sa main.» Ce mode du dire, les Kasena l'assimilent à la langue des signes utilisée pour communiquer avec les personnes atteintes de déficience auditive : "Vugu est sourdmuet [mimaa]. » De fait, certaines lexies du langage de l'oracle, tels les mouvements du bâton pointant le menton du consultant ("celui qui a la barbe»), les mamelons ("la femme»), le menton puis le dos ("l'ancêtre»), sont identiques à celles employées dans la langue des sourds-muets telle que les Kasena la signent. Écriture sans dépôt, sans inscription, écriture fugace qui ne peut se lire que dans l'instant où elle se compose, le dire de l'oracle se tient, comme les langues signées, dans l'entre-deux de la parole et de l'écriture. Écriture sans trace, la langue de l'oracle est, également, une écriture sonore. De même que les différents signes visuels que la canne dessine, indexe, retranche, s'organisent entre eux et font sens, de même les différents sons que la virole métallique de la canne tire du support qu'elle heurte (pierre, fer, terre) forment système. La chorégraphie du bâton divinatoire sollicite, à égalité, ces deux registres sensoriels majeurs que sont la vision et l'audition, mais elle ne leur alloue pas la même fonction. 

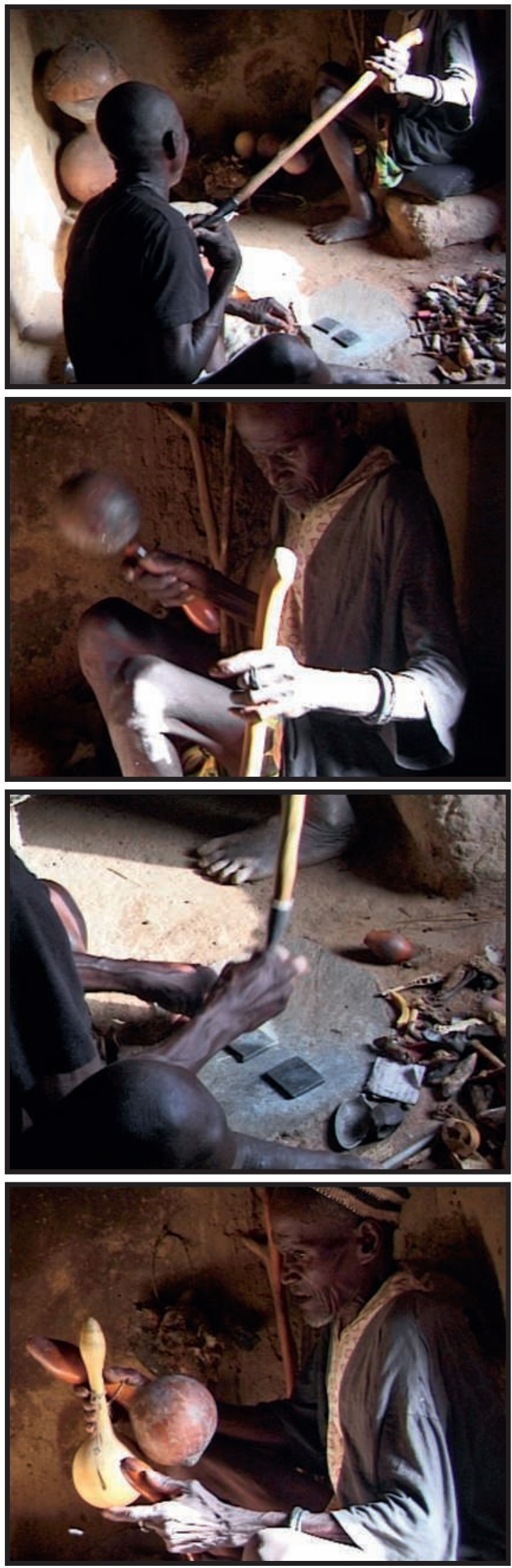

Pour saisir pleinement la portée de ce constat, revenons au procès d'engendrement d'un "dit" de l'oracle et examinons de plus près comment ces trois fonctions de la canne (lexicale, assertorique, syntaxique) s'enchaînent pour produire un énoncé oraculaire. Après une entrée en matière, très brève, où celui qui sait questionner s'assure de l'assentiment de l'oracle à répondre à son interrogation, il va chercher à déterminer pour qui, dans le cercle des présents et des absents, l'oracle va parler. Dans cette introduction, les fonctions assertorique et syntaxique de la canne sont massivement utilisées. Aussitôt qu'il s'est emparé de l'embout ferré de la canne que lui tend le devin, le consultant se met à interroger l'oracle, directement, à haute et intelligible voix, scrutant pour chacun des items en lesquels il décompose sa question l'endroit que vient heurter la virole : l'une des plaquettes en fer, le socle en pierre ou la terre, chacun de ces points d'impact correspondant à une réponse unique (respectivement affirmative, négative et sans objet). Il s'assure de la sorte de la pertinence des différentes unités minimales sur lesquelles porte la "réponse ", avant de chercher à en établir la vérité par la procédure complexe décrite plus haut. La parole du consultant et les mouvements du bâton prennent ici la forme d'un «dialogue» : les sons que tire la virole en fer des plaquettes en métal, de la pierre ou du sol en terre «répondent » à la voix du consultant; ce qui crée au fil de la consultation, où le procédé est récurrent, la représentation d'un dit oraculaire fait d'une nappe sonore minutieusement martelée, ciselée à même les plaquettes en fer. En contrepoint, le murmure du devin vient tantôt redoubler la question du consultant (" où allons-nous, vuru? »), tantôt soutenir le dire de l'oracle («je n'ai pas parlé»). Dans ces préambules, la fonction lexicale de la canne est peu sollicitée, les objets déversés en tas aux pieds du devin ne sont pas utilisés. Leur rôle ne commence que dans le temps qui suit immédiatement ces préliminaires, temps qui s'ouvre avec la question inaugurale : "Qu'y a-t-il [avec cette personne]?» Aussitôt qu'elle a été posée, le régime d'énonciation change. Le consultant abandonne la posture du questionneur, où sa parole précédait les mouvements de la canne, pour celle du scrutateur : il suit attentivement du regard les déplacements du bâton dont il tient toujours l'embout ferré et le devin l'autre extrémité, et les transpose en langage articulé, les met en mots dans le moment même où ils sont produits, l'un après l'autre, sans l'aide du devin s'il est passé maitre dans l'art de questionner, à la suite de ce dernier s'il est encore novice.

Les objets, les traits que dessine la canne dans la poussière du sol, les parties du corps du consultant comme les lieux de l'espace sont autant de signes visuels qui, à l'instant même où ils ont été sélectionnés, déclenchent un acte 
de parole, la production d'un énoncé toujours très bref, du type «je vois... », dans lequel le lexème central est le signe ainsi cueilli à même la réalité. Comme il a déjà été dit, le sens que va recevoir la chose dépend d'un code, mais également de son point d'insertion dans la chaîne des précédentes sélections faites par le bâton. Donnons-en un bref exemple. Le mouvement de l'embout ferré pointant le dessous du mur signifie, dans tel énoncé de l'oracle, «maitre de maison » (qui est censé, après son décès, se tenir "sous les murs ") parce qu'il s'insère dans une suite de "gestes" où ont été successivement sélectionnés le dos du consultant ("l'ancêtre") et son menton ("le vieux»). Dans un autre énoncé, construit par les mêmes protagonistes au cours d'une même séance, ce mouvement pointant le dessous du mur prendra le sens de "Terre", l'instance, parce que cette fois il s'insère dans une chaîne de "signes " qui sont tous relatifs à des matières sacrificielles et à Soleil-Dieu. Autrement dit, l'encodage des objets ne se réduit pas à un simple cryptage, avec un rapport fixe et univoque entre le signifiant et son signifié, mais il repose sur un système où le sens se construit selon des règles qu'on pourrait qualifier de grammaticales, l'ordre dans lequel sont produits les déplacements de la canne l'emportant sur la symbolique des objets. Le langage de la divination par le bâton est de la sorte suffisamment élaboré pour autoriser la description de réalités complexes en des termes entendus par tous, très éloignés de la formulation de sentences sibyllines qu'il reviendrait à des spécialistes d'interpréter.

Les mouvements de la canne et les paroles du consultant s'enchaînent très rapidement pour fournir les premiers mots du texte de l'oracle. C'est la phase initiale. Par contraste avec la courte séquence introductive (mais aussi avec celle qui va suivre), où la voix du consultant et le son métallique de la virole ne cessent de se croiser, cette première phase se caractérise par un silence relatif. À ce stade, en effet, seule la fonction lexicale de la canne est utilisée : c'est le temps de la langue signée, celui où l'oracle donne à voir «ce qu'il y a dans la main de la personne». Son terme est marqué par la toute première forme de ponctuation dont use le langage du bâton, qui associe une formule énoncée par le consultant : "c'est cela que j'ai vu» à un geste spécifique (le balayement de la paume du consultant, répété par trois fois par la canne divinatoire). À cette première partie succède une deuxième où le consultant, tout en cherchant à s'assurer de la vérité des différents lexèmes dégagés dans la phase précédente, les intègre dans une trame discursive plus explicite, plus développée. Comme au temps du prologue, on est revenu à un régime d'énonciation où le consultant questionne et où la canne « répond " de façon sonore, en heurtant les plaquettes en métal, le socle en pierre ou le sol. Cette deuxième division du texte de l'oracle est à son tour rythmée par une "ponctuation " qui associe la formule "c'est cela que tu as dit " à une modalité de frappe très particulière, la virole choquant successivement l'une puis l'autre des plaquettes de métal avant de revenir frapper la première et d'achever son mouvement par un coup amorti contre le sol ${ }^{16}$. Vient ensuite une troisième partie, de même facture, dans laquelle le consultant poursuit son interrogation en dépliant les questions afin d'obtenir plus de précisions sur le lieu, le temps, l'instance destinataire, l'officiant, le contenu d'une prière pour le rite sacrificiel qui aura été notifié dès la première phase. C'est, pour ainsi dire, la phase de "l'enquête». Chaque fois qu'il sera parvenu à établir correctement une réponse, le consultant, en s'aidant des objets déplacés sur la table de consultation, reprendra tout ou partie du texte de l'oracle déjà établi pour y insérer le nouvel élément trouvé. Il marquera la fin de chacune de ces reprises de la même

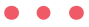

16. Cette série de trois coups alternés (fonction syntaxique) doit être distinguée de celle des trois coups portés sur un même palet (fonction assertorique). Son code en est le suivant : le premier coup « dit »la vérité, le deuxième, le mensonge, le troisième la « vraie vérité » (ku jigi cıga, littéralement «cela saisit le vrai », est un tour de langage qui marque l'intensivité, semblable à notre expression populaire «vrai de vrai »). 


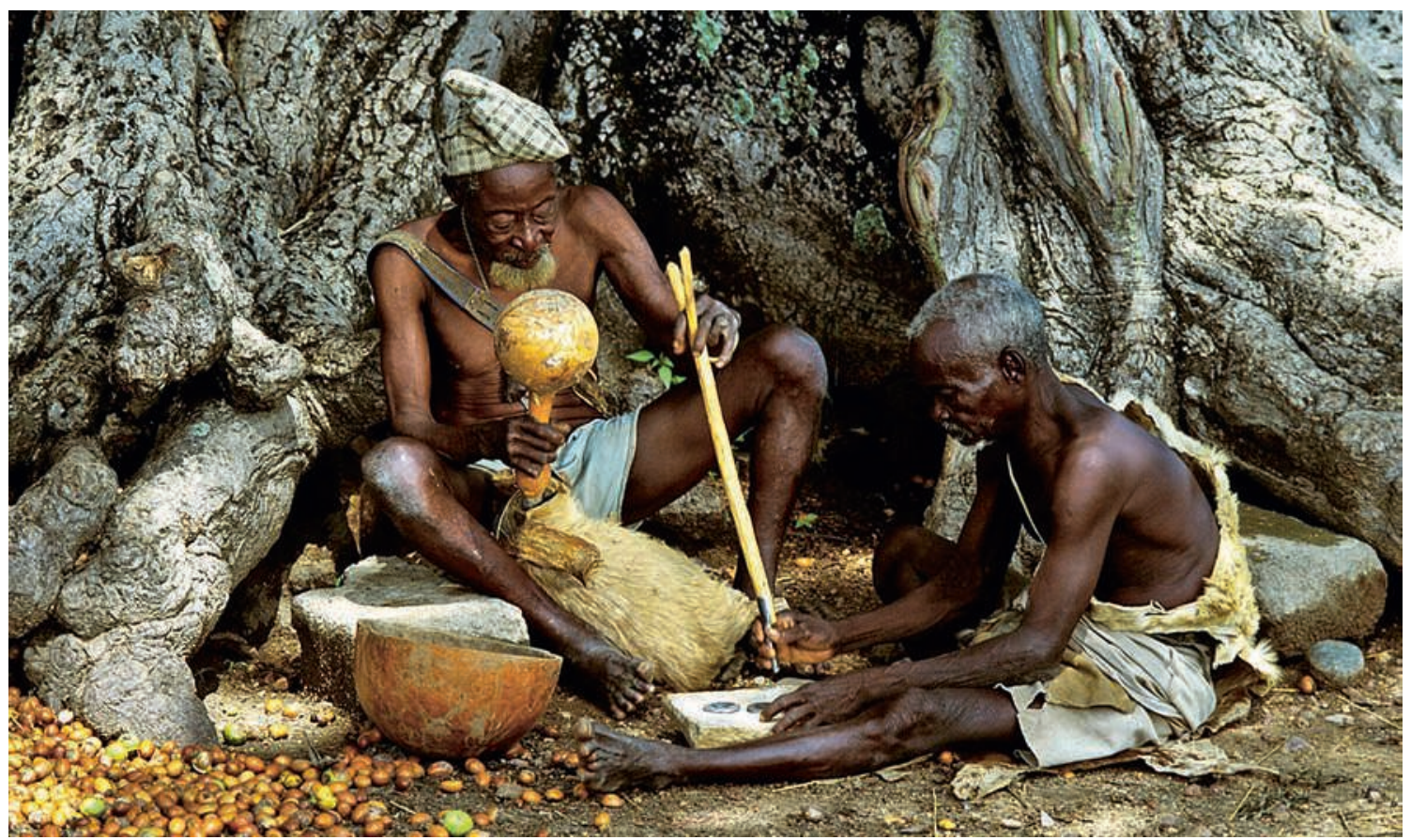

Fig. 7 Cérémonie de divination par le bâton chez les Kasena du Ghana @ Carol Beckwith/Angela Fisher.

façon qu'il a "ponctué " la deuxième phase de son questionnement. Une ultime combinaison fixe de formules ("Qu'y a-t-il à ajouter? »/« Je n'ai pas parlé »/« Nous acceptons ") et de mouvements de la canne clôt le processus, mettant une sorte de point final au texte de l'énoncé oraculaire (une séance en comporte toujours plusieurs).

À propos de formes proches de la divination par le bâton pratiquées par des populations voisines des Kasena, plusieurs auteurs (Fortes, Mendonsa, Sylvie Fainzang) ont cherché à construire l'hypothèse selon laquelle la première phase de la constitution d'un oracle correspondait à la formulation par le devin d'un diagnostic de la situation. La trame suffisamment lâche du constat permettrait au consultant, lors de la deuxième phase, de se l'approprier au terme d'une série d'interrogations qui auraient pour visée de concrétiser le constat, de l'envisager à l'aune de sa situation ou des informations qu'il possède sur la personne concernée par l'oracle. Cette interprétation ne résiste pas aux faits kasena rapportés ici. On lui objectera tout d'abord que le consultant passé maître dans l'art de questionner n'a aucun besoin d'un savoir particulier que détiendrait seul le devin, y compris dans le cas de figure où celui qui consulte est encore novice. D'un point de vue formel, cette situation particulière d'apprentissage pourrait certes laisser penser qu'une telle bipartition du travail existe, car on y entend le devin mettre le premier en mots les objets et les gestes sélectionnés à la pointe de la canne, le consultant se contentant de répéter après lui. Toutefois, l'examen du mode sur lequel le devin donne sa «lecture » empêche de poursuivre dans cette voie. «Estce que tu n'as pas vu cette chose? ", demande le devin au consultant à chaque item sélectionné par la canne divinatoire. Et ce dernier de reprendre «est-ce que je n'ai pas vu cette chose?». En ne recourant jamais au "je " à l'inverse de ce que fait systématiquement celui qui sait questionner - et en laissant à chaque fois le 
novice se repositionner comme sujet de l'énoncé, le devin indique que, même en ce temps de l'apprentissage, c'est le consultant qui occupe la position de «lecteur " des mots de la langue signée que parle l'oracle. Bien qu'il comprenne ce langage signé qu'il produit avec le consultant, le devin semble chercher à s'effacer de la place de seul interprète pour laisser le consultant comme face à face avec l'oracle. La seconde objection s'inscrit dans le prolongement de la première. Ainsi qu'il a été dit, les deux moments structurants dans la fabrication du texte de l'oracle sont respectivement démarqués par les formules : "c'est cela que j'ai vu »/« c'est cela que tu as dit ", dont le consultant est le seul et unique énonciateur. Ce double constat oblige donc à s'écarter d'une lecture qui oppose la première à la deuxième phase comme un constat impersonnel fait par un tiers à sa reformulation par la personne concernée en regard d'éléments qu'elle seule connaît.

Le tas d'objets jeté hors de la besace du devin est un condensé de l'existant, il s'offre comme un miroir du monde. Devin et consultant lisent, à la pointe de la canne divinatoire, les signes muets qu'y a déposés l'oracle puis l'écoutent donner son accord martelé et sonore aux assertions qu'ils en tirent. Les gestes techniques guident strictement les mots du consultant, aussi strictement que le fait l'écriture, mais en renversant la relation habituelle entre parole humaine et écriture. Éphémère comme les pas d'une danse, l'écriture sonore du bâton n'est pas soumise au langage articulé des hommes, elle ne le transcrit ni ne le révèle : elle le suscite; elle rend visible le dit silencieux de l'oracle et le consultant l'épelle. Il n'est donné à personne, fût-ce à un devin, il faut y insister, de pouvoir produire seul un dit de l'oracle. Pour " voir » et « entendre » le dit de l'oracle, il est impératif que la canne soit tenue à chacune de ses extrémités, comme si les protagonistes de l'acte divinatoire étaient «les mains » de ce corps à l'image duquel est construit le dispositif divinatoire (Liberski-Bagnoud 2011). Ils ont en charge, ensemble, d'intercepter au travers de leur propre corps cette écriture visuelle et sonore qu'est le mode du dire oraculaire, à la fois langue signée et percussions rythmiques. Surgissant d'un lieu minutieusement construit au début de chaque consultation, physiquement façonnée au travers du corps-à-corps auquel se livrent devin et consultant, la langue de l'oracle, par son mode même de production, permet ainsi d'élaborer un texte sans auteur, texte que (re)construit minutieusement, mot après mot, phrase après phrase, le consultant, seul ou avec l'aide du devin. Entre la première phase du processus de fabrication du texte de l'oracle et les autres, il y a à l'évidence une différence, qui ne se laisse pas rabattre aisément sur la distinction habituellement faite entre diagnostic et prescription, général et particulier, abstrait et concret. Le fil suivi ici a permis d'entrevoir que ce qui distingue et lie les différents moments dans la constitution d'un «dit» de l'oracle est de même nature que ce qui distingue et lie, dans le registre de la logique discursive, le terme et la proposition, l'idée et le jugement ou, pour prendre le registre du texte, revenu à son sens originel de textus (ce qui est tramé, tissé), la chaîne et le motif enlacés par le fil de la narration.

CNRS-CEMAf

danouta.bagnoud@wanadoo.fr

mots clés / keywords : divination par le bâton // divination by the stick - Afrique de l'Ouest // Western Africa • aire voltaïque // voltaic area • langue des signes // signs language • danse // dance • écriture // writing • corps // body • texte // text. 


\section{Bibliographie}

\section{Adler, Alfred et Zempleni, Andras}

1972 Le Bâton de l'aveugle. Divination, maladie et pouvoir chez les Moundang du Tchad. Paris, Hermann.

\section{BARTHES, Roland}

2000 Le Plaisir du texte, précédé de Variations sur l'écriture, préface de Carlo Ossola. Paris,

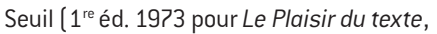
1994 pour Variations sur l'écriture).

\section{BoIsSIÈre, Anne}

2011 «André Schaeffner et les origines corporelles de l'instrument de musique », Methodos, savoirs et textes 11 ; http:// methodos.revues.org/2481.

\section{CARTRY, Michel}

1988 «Le chemin du rite », in Charles Baladier [éd.], Le Grand Atlas des religions. Paris, Encyclopædia Universalis : 296-298 (rééd. in Incidence 6, 2010 : 37-44).

2005 «Eine Orakelschrift/Une écriture divinatoire », in Ruedi Baur (éd.], Das Gesetz und seine visuellen Folgen/La loi et ses conséquences visuelles. Leipzig, Lars Müller Publishers : 402-429.

2009 « De la divination au sacrifice : la métaphore de l'attache », in Michel Cartry (éd.), Architecturer l'invisible. Autels, ligatures, écritures. Turnhout, Brepols [ «Bibliothèque de l'École des hautes études. sciences religieuses 》 CXXXVIII) : 307-360.

\section{Christin, Anne-Marie}

2001 Histoire de l'écriture. De l'idéogramme au multimédia. Paris, Flammarion.

Deleuze, Gilles et Guattari, Félix 1972 Capitalisme et Schizophrénie. L'Anti-EEdipe. Paris, Minuit.

\section{Durand, Jean-Marie}

2008 «Assyriologie », in Cours et travaux du Collège de France. Résumés 2007-2008. L'Annuaire, vol. CVIII : 568; http://annuaire-cdf.revues.org/55.

\section{FAINZANG, Sylvie}

1986 L'Intérieur des choses.

Maladie, divination et reproduction sociale chez les Bissa du Burkina. Paris, L'Harmattan.

\section{ForTes, Meyer}

1987 « Religious premisses and logical technique in the divinatory rituel », in Jack Goody (éd.), Religion, morality and the person. Essays on Tallensi religion. Cambridge, Cambridge University Press : 1-21

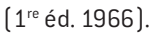

\section{Fraenkel, Béatrice}

2007 «Actes d'écriture : quand écrire c'est faire », Langage et société 3-4(121-122) : 101-112.

\section{JAULIN, Robert}

1966 La Géomancie, analyse formelle. Paris, Mouton.

\section{Legendre, Pierre}

2001 De la société comme texte. Paris, Fayard.

\section{Leroi-Gourhan, André}

1964 Le Geste et la Parole. Technique et langage. Paris, Albin Michel.

\section{Liberski-BAgnoud, Danouta}

2010 « L'espace du dire oraculaire. Aperçu comparatif sur la fabrique d'un lieu d'où peut surgir une parole vraie », Incidence 6 . Paris, Éditions du Félin.

2011 « Le sac du devin kasena et de quelques autres », in Christian Jacob (éd.), Les Lieux du savoir. Les mains de l'intellect, t. II. Paris, Albin Michel.

\section{Mauss, Marcel}

1980 Sociologie et anthropologie. Paris, Presses Universitaires de France

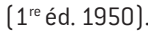

Mendonsa, Eugene

1982 The Politics of divination. Berkeley, University of California Press.

RATTRAY, captain Robert Sutherland 1969 The Tribes of the Ashanti Hinterland.

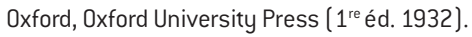

\section{Retel-Laurentin, Anne}

1974 «La force de la parole. Nzakara. Afrique », in Jean-Pierre Vernant 1974 : 285-319.

\section{SCHAEFFNER, André}

1995 Origine des instruments de musique. Introduction ethnologique à l'histoire de la musique instrumentale. Paris, Éditions de l'École des hautes études en sciences sociales

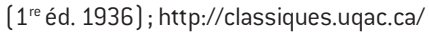
contemporains $/ \mathrm{html}$.

Société internationale de linguistique de Pô

19891000 mots kasim-français. Ouagadougou, Société internationale de linguistique.

Vernant, Jean Pierre et al. 1974 Divination et rationalité. Paris, Seuil. 


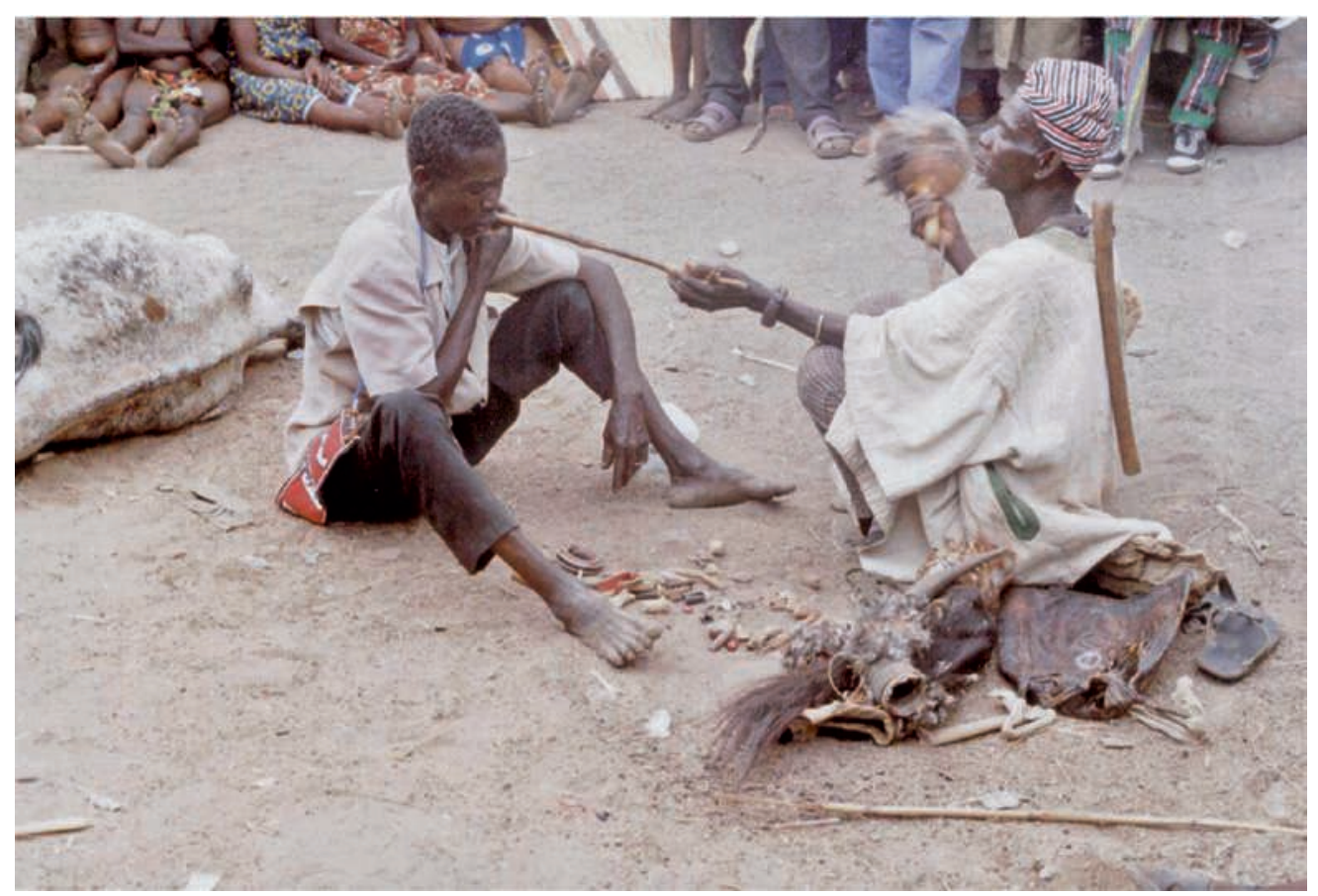

Fig. 9 Séance de divination nankana (ninkarse) lors d'une cérémonie de funérailles, 1997. Photo Urs et Idda Niggli.

\section{Résumé / Abstract}

Danouta Liberski-Bagnoud, La chorégraphie du bâton divinatoire comme écriture sonore au Burkina Faso - À travers l'analyse d'une pratique divinatoire ouest-africaine, dite «par le bâton», cet article aborde la question de la part respective des gestes techniques et de la parole dans la fabrication d'un énoncé oraculaire. $Y$ a-t-il un rapport d'implication mutuelle entre la main qui produit des perceptions visuelles et sonores et les séquences discursives où sont énoncés les mots de l'oracle? L'auteur avance l'hypothèse que l'étrange corps-à-corps du devin et du consultant met en œuvre une forme d'écriture en acte, au sens où en parle Roland Barthes, ainsi que des faits de lecture. Ce que permet ce type de technique divinatoire, c'est autant la transposition en langage clair de signes obtus captés au lieu de l'origine que l'acte de construction d'un texte dont ni le devin ni le consultant ne sont à proprement parler l'auteur.
Danouta Liberski-Bagnoud, The dancing choreography of the divinatory stick as acoustic writing in Burkina Faso - Through the analysis of a Western African divinatory practice called "divination by the stick", this paper addresses the question of the respective part of technical gestures and of language in the creation of a prophetic statement. Is there a relationship of mutual implication between the hand which produces visual and acoustic perceptions and the discursive sequences where the words of the oracle are expressed? The author tests the hypothesis that the divinatory practices expressed by the strange hand-to-hand gestures performed together by the soothsayer and the consultant, implement some form of writing through acts, as defined by Roland Barthes, as well as facts of reading. What is accomplished by this kind of divinatory techniques is as much the transposition of obtuse signs that emerge from the place of origin into a clear language, as the construction of a text of which neither the diviner nor the consultant are, properly speaking, the author. 